\title{
Energetic particles in magnetotail reconnection
}

\author{
Ivy Bo Peng ${ }^{1} \dagger$, Juris Vencels ${ }^{1}$, Giovanni Lapenta ${ }^{2}$, \\ Andrey Divin ${ }^{3}$, Andris Vaivads ${ }^{3}$, Erwin Laure ${ }^{1}$ \\ and Stefano Markidis ${ }^{1}$

\footnotetext{
${ }^{1}$ High Performance Computing and Visualization Department (HPCViz), KTH Royal Institute of Technology, Teknikringen 14, Stockholm 100 44, Sweden

${ }^{2}$ Department of Mathematics, Centre for Mathematical Plasma Astrophysics (CmPA), KU Leuven, Celestijnenlaan 200B, bus 2400 B-3001 Leuven, Belgium Polacksbacken, SE-751 21, Uppsala, Sweden
} \\ ${ }^{3}$ Disciplinary Domain of Science and Technology, Swedish Institute of Space Physics, Uppsala Division,
}

(Received 17 June 2014; revised 22 October 2014; accepted 24 October 2014; first published online 4 December 2014)

We carried out a 3D fully kinetic simulation of Earth's magnetotail magnetic reconnection to study the dynamics of energetic particles. We developed and implemented a new relativistic particle mover in iPIC3D, an implicit Particle-inCell code, to correctly model the dynamics of energetic particles. Before the onset of magnetic reconnection, energetic electrons are found localized close to current sheet and accelerated by lower hybrid drift instability. During magnetic reconnection, energetic particles are found in the reconnection region along the $x$-line and in the separatrices region. The energetic electrons are first present in localized stripes of the separatrices and finally cover all the separatrix surfaces. Along the separatrices, regions with strong electron deceleration are found. In the reconnection region, two categories of electron trajectory are identified. First, part of the electrons are trapped in the reconnection region, bouncing a few times between the outflow jets. Second, part of the electrons pass the reconnection region without being trapped. Different from electrons, energetic ions are localized on the reconnection fronts of the outflow jets.

\section{Introduction}

Magnetic reconnection is a fundamental physical process that results in the topological change of magnetic field, plasma heating, and acceleration of energetic particles (Birn and Priest 2007; Priest and Forbes 2007). It occurs almost everywhere where strong currents flow within plasma, e.g. in space, astrophysical and fusion device plasmas. In Earth magnetosphere, magnetic reconnection occurs in the magnetotail (Nagai et al. 2001; Øieroset et al. 2002a; Retinò et al. 2008; Birn et al. 2012; Nagai et al. 2013) and in the dayside magnetopause (Sonnerup 1974; Daughton et al. 2011). It regulates the exchange of energy and momentum between the solar wind and the inner magnetosphere. Magnetic reconnection accelerates particles, converting magnetic field energy into kinetic energy of the particles (Kivelson and Russell 1995). This paper focuses on the study of the dynamics of energetic particles during the magnetic reconnection in an antiparallel magnetic field set-up that mimics the Earth's magnetotail configuration with no guide field.

The scientific question that motivates this study is to understand the dynamics and acceleration mechanisms of energetic particles in magnetotail magnetic reconnection.

$\dagger$ Email address for correspondence: bopeng@kth.se 
Acceleration mechanisms in 3D kinetic simulations are less studied than in 2D, but are important for understanding how plasma is accelerated to high energies and which physical mechanisms regulate the dynamics of energetic particles during magnetotail magnetic reconnection (Birn and Priest 2007; Priest and Forbes 2007; Birn et al. 2012). Our simulation results are in agreement with the results from previous simulations in 2D geometry and reduced physical models, corroborating previous studies in acceleration mechanisms in magnetic reconnection (Hoshino et al. 2001; Egedal et al. 2005).

In the context of this study, we define high energetic particles as those particles whose velocity is substantially larger than the initial thermal velocity. To answer the scientific question, we performed fully kinetic 3D Particle-in-Cell simulations, saving information of particle trajectories at high temporal frequency. The main challenge of this work is to analyze the large volume of output data saved during the simulations to track down those particles that reached high energy and to reconstruct high-resolution trajectories of these particles.

In order to study the trajectory of relativistic particles, we implemented a new relativistic mover in the $\mathrm{iPIC} 3 \mathrm{D}$ code. This particle mover uses a predictor-corrector technique (Press 2007) to solve the relativistic equation of particle motion. Other formulations of relativistic mover are described in Birdsall and Langdon (2004), Noguchi et al. (2007) and Vay (2008). Previous studies of particle trajectories in magnetic reconnection were carried out either in 2D geometry (Hoshino et al. 2001; Ricci et al. 2003) or used test particles in combination with MHD and PIC simulations (Divin et al. 2010; Ashour-Abdalla et al. 2011). In our work, we look into the 3D trajectories calculated with a fully kinetic self-consistent Particle-in-Cell code.

Several acceleration mechanisms are present during magnetic reconnection. Depending on the location of electrons, they can be accelerated near an X-type neutral region (Øieroset et al. 2002b; Sharma et al. 2008) or around the magnetic field piled-up region (Vapirev et al. 2013; Lapenta et al. 2014). Particles can be accelerated by the inductive reconnection electric field and by electrostatic fields along the separatrices (Hoshino et al. 2001; Hoshino 2005; Birn and Priest 2007). The Speiser motion is described as alternating particle motion between magnetotail lobes. While particles are trapped in magnetic field, the reconnection electric field accelerates them (Speiser 1965).

The paper is organized as follows. The second section presents the development of relativistic mover for the iPIC3D code. The third section describes the simulation parameters and the initial setup to mimic magnetic reconnection in magnetotail. The fourth section reports the simulation results of magnetic reconnection in magnetotail: the evolution of magnetic reconnection is presented first, then the positions and trajectories of energetic electron and ion are shown as well as the velocity distribution of energetic electrons in different cycles. The fifth section concludes the paper, summarizing the results and discussing the validity of the presented simulation.

\section{Relativistic particle mover}

In this study, the Vlasov and Maxwell equations are numerically solved using the Particle-in-Cell method. Electrons and ions are mimicked by a reduced number of computational particles that move in a self-consistent electro-magnetic field (Hockney and Eastwood 1988; Birdsall and Langdon 2004). We run simulations with the implicit Particle-in-Cell code iPIC3D (Markidis et al. 2010) that solves the classical equation of particle motion in particle mover. To study the dynamics 
of energetic particles and to correctly model their trajectories, we implemented a new particle mover that solves the relativistic equation of particle motion. Each computational particle is characterized by a position $\mathbf{x}_{p}$ and a velocity $\mathbf{v}_{p}$, whose evolution is described by the relativistic equation of motion (here in CGS system):

$$
\left\{\begin{array}{l}
\frac{d \mathbf{x}_{p}}{d t}=\mathbf{v}_{p} \\
\frac{d\left(m_{s} \gamma \mathbf{v}_{p}\right)}{d t}=q_{s}\left(\mathbf{E}_{p}+\frac{\mathbf{v}_{p}}{c} \times \mathbf{B}_{p}\right),
\end{array}\right.
$$

where $\gamma$ is the Lorentz factor, defined as $\gamma=1 / \sqrt{1-\mathbf{v}_{p}^{2} / c^{2}} \cdot m_{s}$ and $q_{s}$ are the mass and the charge of the species $s$, and $\mathbf{E}_{p}, \mathbf{B}_{p}$ are the electric and magnetic fields acting on the particle. The relativistic equation of motion is first discretized in time and then integrated numerically (Noguchi et al. 2007). The relativistic equation of motion is discretized in time following the midpoint rule as follows:

$$
\left\{\begin{array}{l}
\mathbf{x}_{p}^{n+1}=\mathbf{x}_{p}^{n}+\mathbf{v}_{p}^{n+1 / 2} \Delta t \\
\mathbf{u}_{p}^{n+1}=\mathbf{u}_{p}^{n}+\left(\frac{q}{m}\right)_{s} \Delta t\left(\mathbf{E}_{p}^{n+1}+\frac{\mathbf{v}_{p}^{n+1 / 2} \times \mathbf{B}_{p}^{n+1}}{c}\right),
\end{array}\right.
$$

where $\gamma \mathbf{v}_{p}=\mathbf{u}_{p}$ and the average velocity is defined as

$$
\overline{\mathbf{v}}_{p}=\mathbf{v}_{p}^{n+1 / 2}=\frac{\mathbf{u}^{n+1}+\mathbf{u}^{n}}{\gamma^{n+1}+\gamma^{n}} .
$$

We rewrite the second equation in (2.2), using (2.3):

$$
\mathbf{v}_{p}^{n+1 / 2}=\frac{2 \mathbf{u}_{p}^{n}}{\gamma^{n+1}+\gamma^{n}}+\frac{q_{s} \Delta t}{m_{s}\left(\gamma^{n+1}+\gamma^{n}\right)}\left(\mathbf{E}_{p}^{n+1}+\frac{\mathbf{v}_{p}^{n+1 / 2} \times \mathbf{B}_{p}^{n+1}}{c}\right) .
$$

The cross product term can be expressed as:

$$
\begin{aligned}
\mathbf{v}_{p}^{n+1 / 2} \times \mathbf{B}_{p}^{n+1}= & \frac{2 \mathbf{u}_{p}^{n} \times \mathbf{B}_{p}^{n+1}}{\gamma^{n+1}+\gamma^{n}}+\frac{q_{s} \Delta t}{m_{s}\left(\gamma^{n+1}+\gamma^{n}\right)} \mathbf{E}_{p}^{n+1} \times \mathbf{B}_{p}^{n+1} \\
& +\frac{q_{s} \Delta t}{m_{s} c\left(\gamma^{n+1}+\gamma^{n}\right)}\left(\left(\mathbf{v}_{p}^{n+1 / 2} \cdot \mathbf{B}_{p}^{n+1}\right) \mathbf{B}_{p}^{n+1}-\left(\mathbf{B}_{p}^{n+1}\right)^{2} \mathbf{v}_{p}^{n+1 / 2}\right) .
\end{aligned}
$$

Similarly, the dot product term can be expressed as:

$$
\mathbf{v}_{p}^{n+1 / 2} \cdot \mathbf{B}_{p}^{n+1}=\frac{2 \mathbf{u}_{p}^{n} \cdot \mathbf{B}_{p}^{n+1}}{\gamma^{n+1}+\gamma^{n}}+\frac{q_{s} \Delta t}{m_{s}\left(\gamma^{n+1}+\gamma^{n}\right)} \mathbf{E}_{p}^{n+1} \cdot \mathbf{B}_{p}^{n+1} .
$$

After inserting (2.5) and (2.6) in (2.4), it is possible to find an expression for $\overline{\mathbf{v}}_{p}=\mathbf{v}_{p}^{n+1 / 2}$ :

$$
\begin{aligned}
& \tilde{\mathbf{u}}_{p}=\gamma^{n} \mathbf{v}_{p}^{n}+\frac{q_{s}}{m_{s}} \frac{\Delta t}{2} \mathbf{E}_{p}^{n+1}, \\
& \overline{\mathbf{v}}_{p}=\frac{\tilde{\mathbf{u}}_{p}+\frac{q_{s}}{m_{s}} \frac{\Delta t}{2 c}\left[\frac{\tilde{\mathbf{u}}_{p}}{\tilde{\gamma}} \times \mathbf{B}_{p}^{n+1}+\frac{q_{s}}{m_{s}} \frac{\Delta t}{2 c}\left(\frac{\tilde{\mathbf{u}}_{p}}{\tilde{\gamma}} \mathbf{B}_{p}^{n+1}\right) \mathbf{B}_{p}^{n+1}\right]}{\tilde{\gamma}\left[1+\left(\frac{q_{s}}{m_{s}} \frac{\Delta t}{2 c \tilde{\gamma}} \mathbf{B}_{p}^{n+1}\right)^{2}\right]} .
\end{aligned}
$$

We note that the only approximation we use to derive this equation is $\tilde{\gamma} \approx$ $\left(\gamma^{n}+\gamma^{n+1}\right) / 2$. Equation (2.8) is solved by a predictor-corrector method with a fixed 
number of iterations. At each predictor-corrector iteration, the field values $\mathbf{E}_{p}, \mathbf{B}_{p}$ are calculated in the position $\mathbf{x}_{p}^{n+1 / 2}=\mathbf{x}_{p}^{n}+\overline{\mathbf{v}}_{p} \Delta t / 2$. There is no guarantee for $\overline{\mathbf{v}}_{p}$ to be less than the speed of light in vacuum after the predictor-corrector iterations. For this reason, we check that $\overline{\mathbf{v}}_{p}<c$ to ensure that $\overline{\mathbf{v}}_{p}$ has a real value. In the case of $\overline{\mathbf{v}}_{p}>c$, the time step is reduced and the sub-cycling technique is used.

From (2.3), we derive $\left(\mathbf{u}^{n+1}\right)^{2}$ and insert the result in $\gamma^{n+1}=\sqrt{1+\left(\mathbf{u}^{n+1}\right)^{2} / c^{2}}$. After a series of algebraic manipulations, the Lorentz factor at time $n+1$ is expressed as:

$$
\gamma^{n+1}=\frac{2\left(\gamma^{n} \overline{\mathbf{v}}_{p}^{2}-\overline{\mathbf{v}}_{p} \mathbf{u}_{p}^{n}\right)+\sqrt{4\left(\gamma^{n} \overline{\mathbf{v}}_{p}^{2}-\overline{\mathbf{v}}_{p} \mathbf{u}_{p}^{n}\right)^{2}+4\left(c^{2}-\overline{\mathbf{v}}_{p}^{2}\right)\left(c^{2}+\gamma^{n} \overline{\mathbf{v}}_{p}^{2}+\left(\mathbf{u}_{p}^{n}\right)^{2}-2 \gamma^{n} \overline{\mathbf{v}}_{p} \mathbf{u}_{p}^{n}\right)}}{2\left(c^{2}-\overline{\mathbf{v}}_{p}^{2}\right)} .
$$

$\overline{\mathbf{v}}_{p}$ is evaluated with the predictor-corrector technique, then the Lorentz factor at time $n+1$ is calculated. Finally, after the predictor-corrector iterations to calculate $\overline{\mathbf{v}}_{p}$, the particle position and velocity are advanced as follows:

$$
\left\{\begin{array}{l}
\mathbf{v}_{p}^{n+1}=\frac{\overline{\mathbf{v}}_{p}\left(\gamma^{n+1}+\gamma^{n}\right)-\gamma^{n} \mathbf{v}_{p}^{n}}{\gamma^{n+1}} \\
\mathbf{x}_{p}^{n+1}=\mathbf{x}_{p}^{n}+\overline{\mathbf{v}}_{p} \Delta t
\end{array}\right.
$$

In this simulation, we chose a typical number of three predictor-corrector iterations for each cycle. Experiments and studies show good convergence and energy conservation at this number of iterations (Lapenta et al. 2006; Markidis et al. 2010).

\section{Simulation set-up}

We carried out a 3D Particle-in-Cell simulation of magnetotail reconnection. We used a typical configuration with a Harris current sheet initially imposed and with no guide field added in the model. We followed the geocentric solar magnetospheric system (GSM) for the simulation (Russell 1971). The $y$ coordinate is taken along the Harris sheet current, while the $x-z$ plane is the reconnection plane. The $x$ direction is taken from the Earth to the Sun in the Earth's magnetosphere. The $y$ and $z$ directions are pointing from East to West and from South to North respectively.

An antiparallel magnetic field, vanishing in the midplane of the simulation domain $\left(z=L_{z} / 2\right)$ (Birn et al. 2001), is initialized in the $x$ direction as:

$$
B_{x}(z)=B_{0} \tanh \left(\frac{z-L_{z} / 2}{\lambda}\right) .
$$

The asymptotic magnetic field $B_{0}$ is $25 n T$. The plasma density profile is initialized as:

$$
n(z)=n_{0} \cosh ^{-2}\left(\frac{z-L_{z} / 2}{\lambda}\right)+n_{b} .
$$

The peak density $n_{0}=0.25$ particle $\mathrm{cm}^{-3}$ is used as the reference density. The background density is $n_{b}=0.1 n_{0}$. The ion inertial length is $d_{i}=c / \omega_{p i}=85 \mathrm{~km}$, where $c$ is the speed of light in vacuum, $\omega_{p i}=\sqrt{4 \pi n_{0} e^{2} / m_{i}}=3523 \mathrm{rad} \mathrm{s}^{-1}$ the ion plasma frequency, $e$ is the elementary charge and $m_{i}$ is the ion mass. The simulation box is $L_{x} \times L_{y} \times L_{z}=1700 \mathrm{~km} \times 850 \mathrm{~km} \times 1276 \mathrm{~km}\left(20 d_{i} \times 10 d_{i} \times 15 d_{i}\right)$. Half-width of the current sheet is $\lambda=42.5 \mathrm{~km}\left(0.5 d_{i}\right)$. To reduce the computational cost of the simulation, an electron mass $m_{e}=m_{i} / 64$ is used.

The particles are initialized with a Maxwellian velocity distribution. The electron thermal velocity is $v_{t h e}=23367 \mathrm{~km} \mathrm{~s}^{-1}(0.0779 \mathrm{c})$. This corresponds to an electron 
energy $3.1 \mathrm{keV}$. The ion temperature is $T_{i}=5 T_{e}$. The electron and ion current sheet drift velocities are $1948 \mathrm{~km} \mathrm{~s}^{-1}(0.0065 \mathrm{c})$ and $9743 \mathrm{~km} \mathrm{~s}^{-1}(0.0325 \mathrm{c})$ respectively. With magnetic field from (3.1), we calculate the total current $\mathbf{J}$ using Ampere's law. After that, we redistributed the current between ions and electrons (electron current is $1 / 5$ of the ion current) as done in previous works (Vapirev et al. 2013; Lapenta et al. 2014).

The simulation time step is $\Delta t=8.5 \times 10^{-5} \mathrm{~s}\left(0.2 \omega_{p i}^{-1}\right)$ and a time period of $0.2 \mathrm{~s}$ $\left(2353 \omega_{p i}^{-1}\right)$ is simulated. The sub-cycling technique is used in the relativistic particle mover with three sub-cycles for both ion and electron so that $\Delta t_{e}=2.8 \times 10^{-5} \mathrm{~s}$ $\left(0.0667 \omega_{p i}^{-1}\right)$. In this simulation set-up, the Alfvén velocity calculated with $B=B_{0}$ and $n=n_{0}$ is $5,846 \mathrm{~km} \mathrm{~s}^{-1}$. The ratio $c / V_{A}=\omega_{p i} / \Omega_{c i}$ results to 51.3 . The electron and ion Larmor gyro-radius are respectively $3 \mathrm{~km}\left(0.035 d_{i}\right)$ and $55 \mathrm{~km}\left(0.647 d_{i}\right)$.

The grid is composed of $256 \times 128 \times 196$ cells. In total, $7 \times 10^{8}$ computational particles are used. Simulations are carried out with the massively parallel implicit Particle-in-Cell iPIC3D code (Markidis et al. 2010), running 1024 MPI processes on Lindgren supercomputer at KTH Royal Institute of Technology. The use of an implicit Particle-in-Cell code allows us to use a smaller number of particles, compared to typical explicit PIC simulations. This is because the total statistical noise is smaller in implicit PIC code than in explicit PIC code (Lapenta et al. 2006). Both field and particle boundaries are periodic in the $x$ and $y$ directions. In the $z$ direction, perfect conductor is used for the field boundaries and reflecting boundary conditions are used for particles.

\section{Simulation results}

In this section, we first describe the macroscopic evolution of the system and then focus on the study of energetic electrons and ions. The Particle-in-Cell simulation shows all the characteristic signatures of magnetic reconnection: the Hall magnetic field shows the quadrupolar structure and a reconnection electric field develops in proximity of the $x$-line.

Figure 1 shows a contourplot of the Hall magnetic field $\left(B_{y}\right)$ on the midplane $y=L_{y} / 2$ and the $y$-component of electric field $\left(E_{y}\right)$ on the midplane $z=L_{z} / 2$ at three different times. $B_{y}$ in the left panel shows the characteristic quadrupolar structure in an expanding pattern from $t=0.08 \mathrm{~s}$ to $t=0.2 \mathrm{~s}$. $E_{y}$ in the right panel shows wave structures (visible as blue and red stripes) that are initially propagating in the $y$ direction at time $t=0.08 \mathrm{~s}$. Their wavelength and intensity values are in good agreement with the previous simulation results of lower-hybrid waves caused by lower-hybrid drift instability (Daughton 2003; Lapenta et al. 2003; Ricci et al. 2005). At time $t=0.17 \mathrm{~s}, E_{y}$ plot shows that the reconnection electric field (an inductive electric field that forms as a result of the temporal change in the magnetic field topology) forms along the $x$-line (a magnetic null line (Finn 2006)) along the $y$ direction at the center of the simulation box. The reconnection electric field reaches approximately values $40 \mathrm{mV} \mathrm{m}^{-1}\left(\approx 0.3 B_{0} c / V_{A}\right)$. We note that the value of the reconnection electric field is in agreement with previous works in dimensionless units, but much higher than observations in SI units. This is due to the reduced ion to electron mass ratio, which results the $c / V_{A}$ ratio to be lower than the value observed in the magnetotail (Lapenta et al. 2010). At time $t=0.2 \mathrm{~s}$, the presence of an instability affecting the reconnection fronts is visible in the plot (Vapirev et al. 2013; Lapenta et al. 2014). By inspecting the temporal evolution of the $E_{y}$ value at 

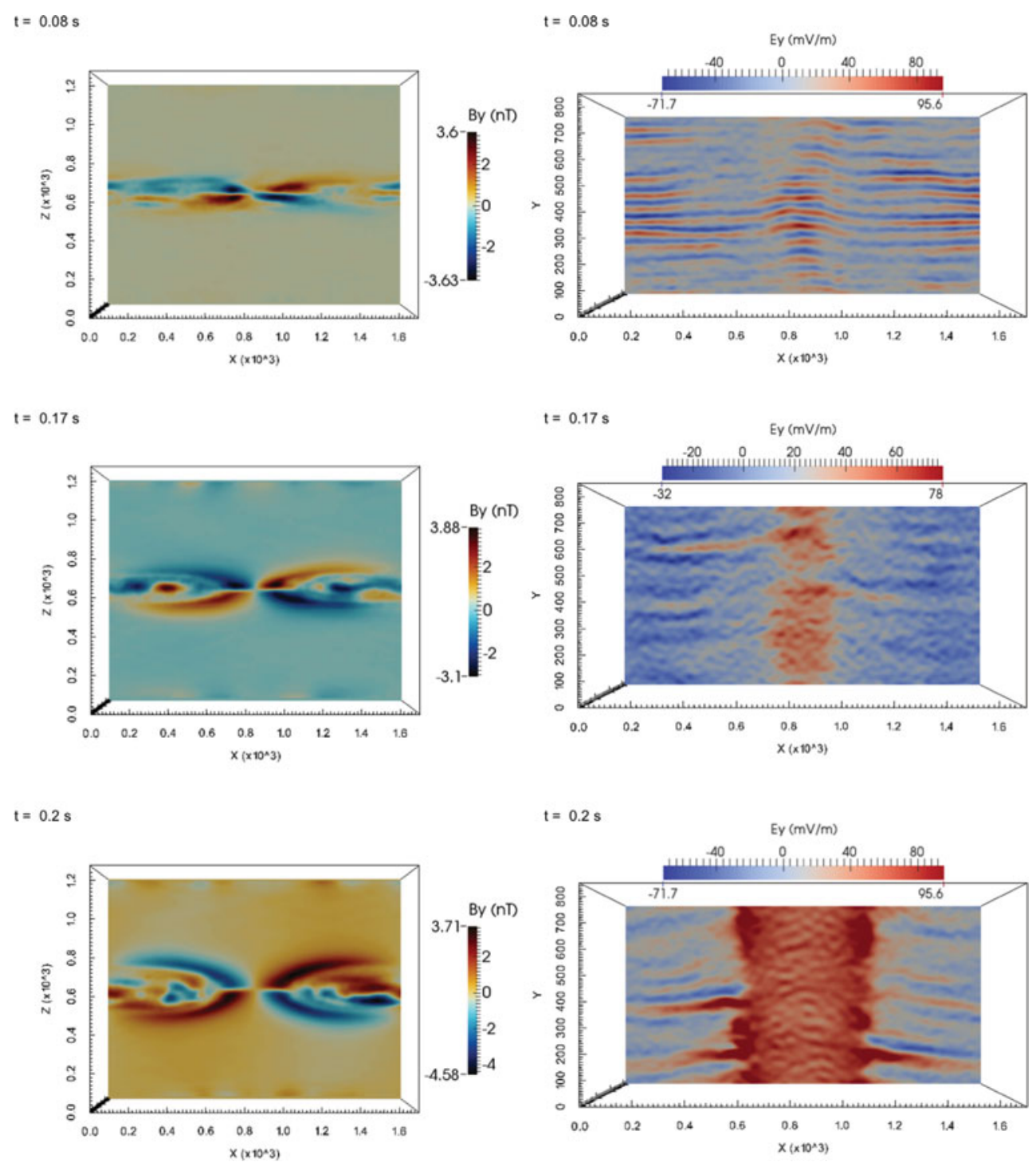

FIGURE 1. Contourplot of the Hall magnetic field $B_{y}$ (left) on the midplane $y=L_{y} / 2$ and the $y$-component of electric field $E_{y}$ (right) on the midplane $z=L_{z} / 2$ at three different times. The Hall magnetic field shows the characteristic quadrupolar structure. $E_{y}$ shows the presence of lower-hybrid drift instability at time $t=0.08 \mathrm{~s}\left(941 \omega_{p i}^{-1}\right)$. The reconnection electric field is clear at time $t=0.17 \mathrm{~s}\left(2000 \omega_{p i}^{-1}\right)$. At time $t=0.2 \mathrm{~s}\left(2353 \omega_{p i}^{-1}\right)$ the instability affecting the reconnection fronts is visible.

the center of the simulation box $\left(x=L_{x} / 2, y=L_{y} / 2, z=L_{z} / 2\right)$ we identified the different stages of magnetic reconnection. The linear phase of magnetic reconnection starts approximately at time $t=0.15 \mathrm{~s}$ and the consequent saturation phase begins at time $t=0.18 \mathrm{~s}$.

As a result of magnetic reconnection, jets exit the $x$-line (Daughton 2003). Figure 2 presents a contourplot of the $x$-component of electron and ion fluid velocities, with superimposed quiver plot of electron and ion bulk fluid velocities on the midplane $z=L_{z} / 2$. The electron jets exiting the $x$-line are visible. Their velocities in the 

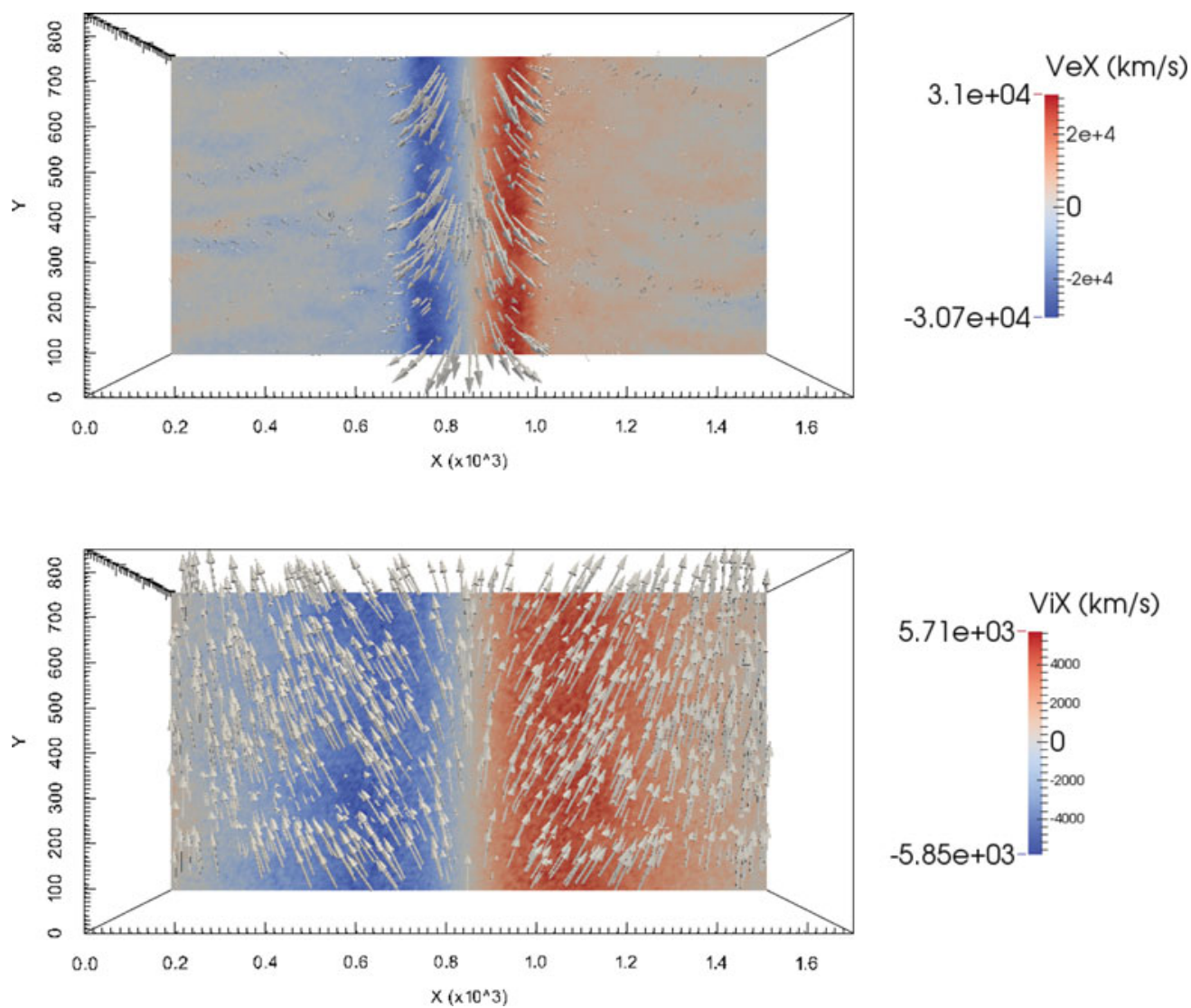

FIGURE 2. Contourplot of the $x$ component of electron (top) and ion (bottom) fluid velocities, with superimposed quiver plot of electron and ion bulk fluid velocities on the midplane $z=L_{z} / 2$ at time $t=0.2 \mathrm{~s}$. The electron jets exiting the $x$-line are visible. Their velocities in the $x$ direction are approximately six Alfvén velocities. Ion jets are broader and have Alfvén velocity. In both cases, the flow is mainly along the $y$ direction (the reconnection electric field direction).

$x$ direction are approximately six times the Alfvén velocity $\left(V_{A}=5846 \mathrm{~km} \mathrm{~s}^{-1}\right)$. Ion jets are broader in space and have an outflow velocity equal to the Alfvén velocity (Birn and Priest 2007; Priest and Forbes 2007). In both cases, by analyzing the quiver plot, it is clear that the flow is mainly along the $y$ direction (the reconnection electric field direction).

\subsection{Energetic electrons}

This paper focuses on the study of the dynamics of energetic particles during magnetotail magnetic reconnection. We chose high energetic particles as those particles whose final velocity is substantially larger than the initial thermal velocity. To identify the electrons with high velocity, we plotted the positions of electrons whose velocities are higher than $54000 \mathrm{~km} \mathrm{~s}^{-1}$ at time $0.08 \mathrm{~s}$ and $67500 \mathrm{~km} \mathrm{~s}^{-1}$ at time $0.17,0.18,0.2 \mathrm{~s}$ in Fig. 3. At time $0.08 \mathrm{~s}$, the signature of the lower hybrid drift instability (Lapenta et al. 2003; Ricci et al. 2005) is visible and consistent with the $E_{y}$ plot in Fig. 1 at the same time step. The lower hybrid drift instability accelerate electrons to higher velocities. At time $0.17 \mathrm{~s}$, the energetic electrons are localized in the reconnection region. At time $0.18 \mathrm{~s}$, energetic electrons can be found in the 

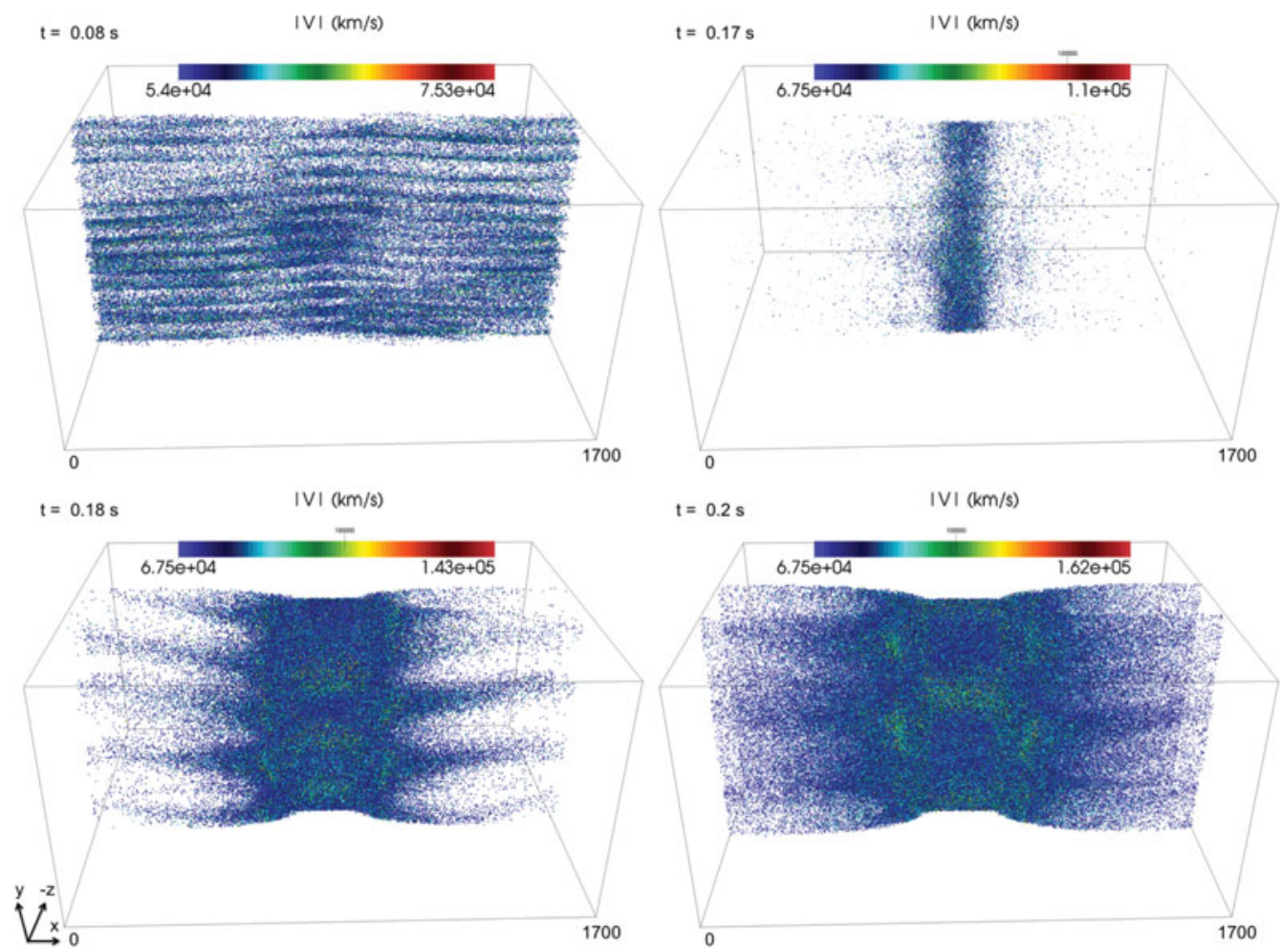

FIGURE 3. Scatter plot of the electrons with $|v|>54000 \mathrm{~km} \mathrm{~s}^{-1}$ at time $0.08 \mathrm{~s}$ and $|v|>67500 \mathrm{~km} \mathrm{~s}^{-1}$ at times $0.17,0.18,0.2 \mathrm{~s}$. The point color represents the speed of particle. During the simulation the maximum electron speed in the system increases in time. At time $0.08 \mathrm{~s}$, high energetic electrons are accelerated by lower-hybrid drift instability along the current sheet, where the lower-hybrid wave is visible and consistent with the first E field in the right panel of Fig. 1. In the following times, high energetic particles are localized in reconnection region and along the separatrices regions.

separatrices area also but only along certain regions with stripe shape. At time $0.2 \mathrm{~s}$ energetic electrons are present in the reconnection region and in the separatrix region.

From inspecting the scatter plots in Fig. 3, it is clear that energetic electrons are localized in a relatively small reconnection region along the $x$-line and along the separatrice surfaces. For this reason, we focused on the study of electron trajectories whose position at the end of the simulation is in proximity of the reconnection region.

Figure 4 shows the trajectories of 4000 randomly chosen electrons ending in the reconnection region. The electron trajectories move along the separatrix surfaces mainly on the $x-z$ plane. The color of the line represents the electron velocity. The regions enclosed in the white dashed boxes, in the separatrices region, shows the abrupt presence of electrons with almost vanishing velocity. This is probably the result of a kinetic instability such as Buneman or two stream instability as reported in previous studies (Lapenta et al. 2011; Divin et al. 2012; Markidis et al. 2012).

When inspecting the trajectories of 4000 electrons whose final position is in the reconnection region, we found two kinds of trajectories. First, a part of the electrons enter the reconnection region drifting along the separatrices and bounce a few times in the reconnection region. These electrons ended trapped in the reconnection region. The trapping time varies for different electrons: the majority of trapped electrons 


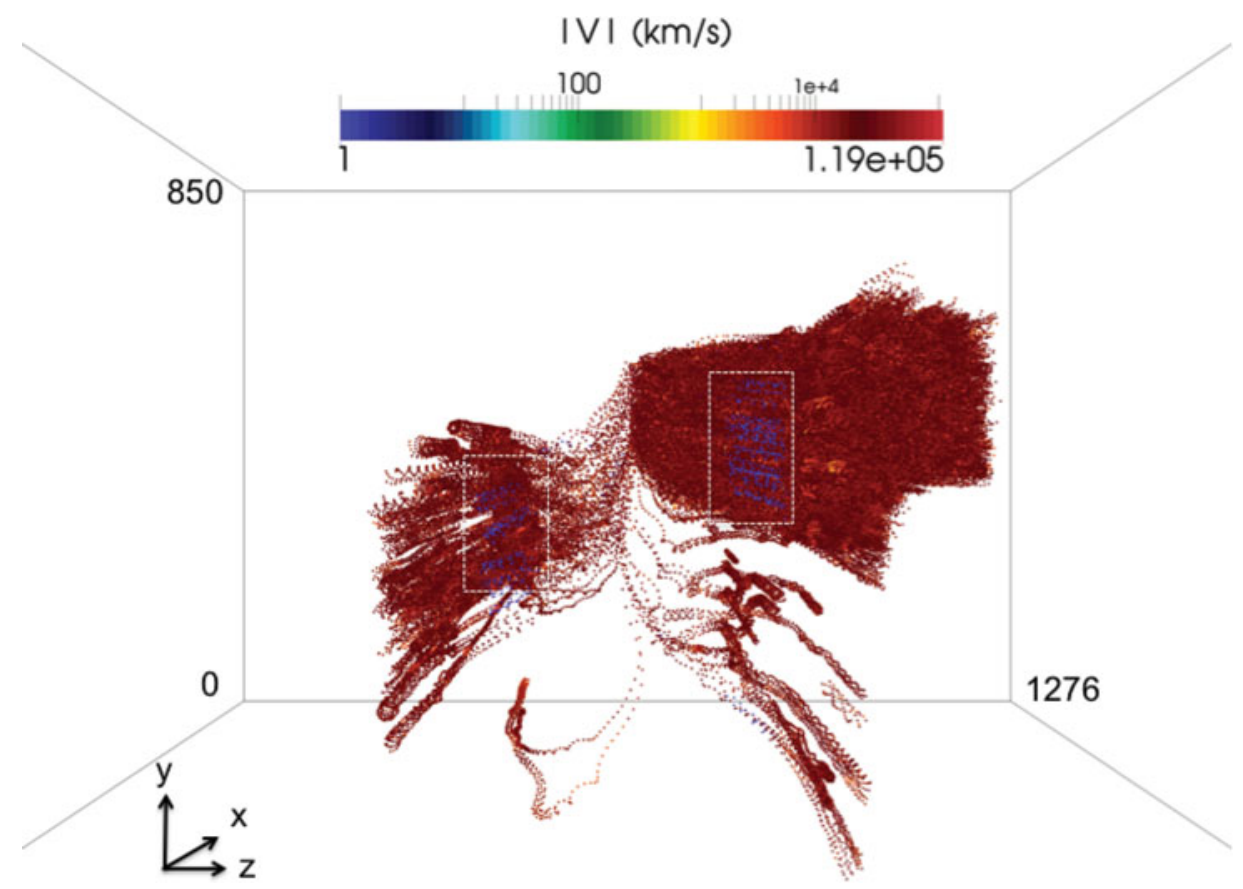

FIGURE 4. Trajectories of 4000 electrons ending up in the reconnection region. Trajectories are along the separatrix surfaces. The regions enclosed in the white dashed boxes shows the presence of electrons with low velocity. This is probably the result of a kinetic instability such as Buneman or two stream instability.

are confined in the reconnection region for the whole simulation time. Simulations covering a longer period of time are needed to determine the trapping time. Figure 5 shows the trajectories of selected electrons that are trapped in the reconnection region. Different colors represent different electron trajectories. A contourplot of $B_{z}$ at time $0.2 \mathrm{~s}$ is superimposed in the plot to show the variation of magnetic field close to the reconnection region.

A second category of electron trajectories can be identified by analyzing Fig. 6 . These electrons pass the reconnection region without being reflected or being trapped.

Figure 7(a) presents the electron velocity distribution function for $|v|>$ $54000 \mathrm{~km} \mathrm{~s}^{-1}$ at times $t=0.17,0.185,0.2 \mathrm{~s}$ in green, blue and red respectively. From this plot, it is clear that the number of relativistic electrons increases during magnetic reconnection. Figure 7(b) shows the trajectories of electrons with $|v|>1 / 2 c$. The electrons with highest velocity are accelerated by the reconnection electric field along the $x$-line (the $y$ direction at the center of the simulation box). Over a total of $3.5 \times 10^{8}$ electrons, we found only 24 electrons $\left(<3.5 \times 10^{-6} \%\right)$ moving faster than $c / 2$. The trajectories of these relativistic electrons are shown in green in Fig. 7(b). These high energetic electrons move along the $y$ direction and are solely accelerated by the reconnection electric field $E_{y}$. It shows that the main mechanism to accelerate electrons to relativistic energies is by reconnection electric field.

\subsection{Energetic ions}

As part of this study, we repeated the same analysis for the high velocity ions present in the magnetic reconnection simulation. We extracted ions with $|v|>26440 \mathrm{~km} \mathrm{~s}^{-1}$ at time $t=0.2 \mathrm{~s}$ and found that the most energetic ions are localized in proximity 


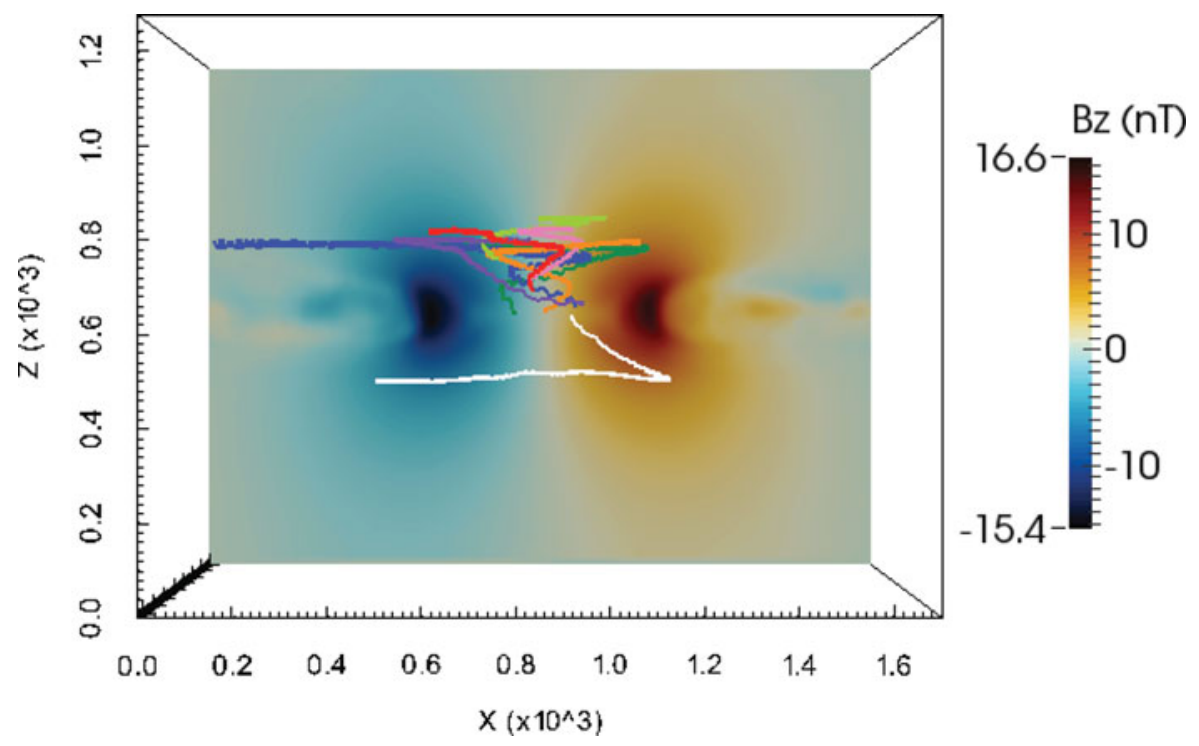

FIGURE 5. Trajectories of trapped electrons in the reconnection region superimposed to the contourplot of $B_{z}$ at time $0.2 \mathrm{~s}\left(2353 \omega_{p i}^{-1}\right)$. The different colors represent different electron trajectories. These electrons bounce a few times in the reconnection region.

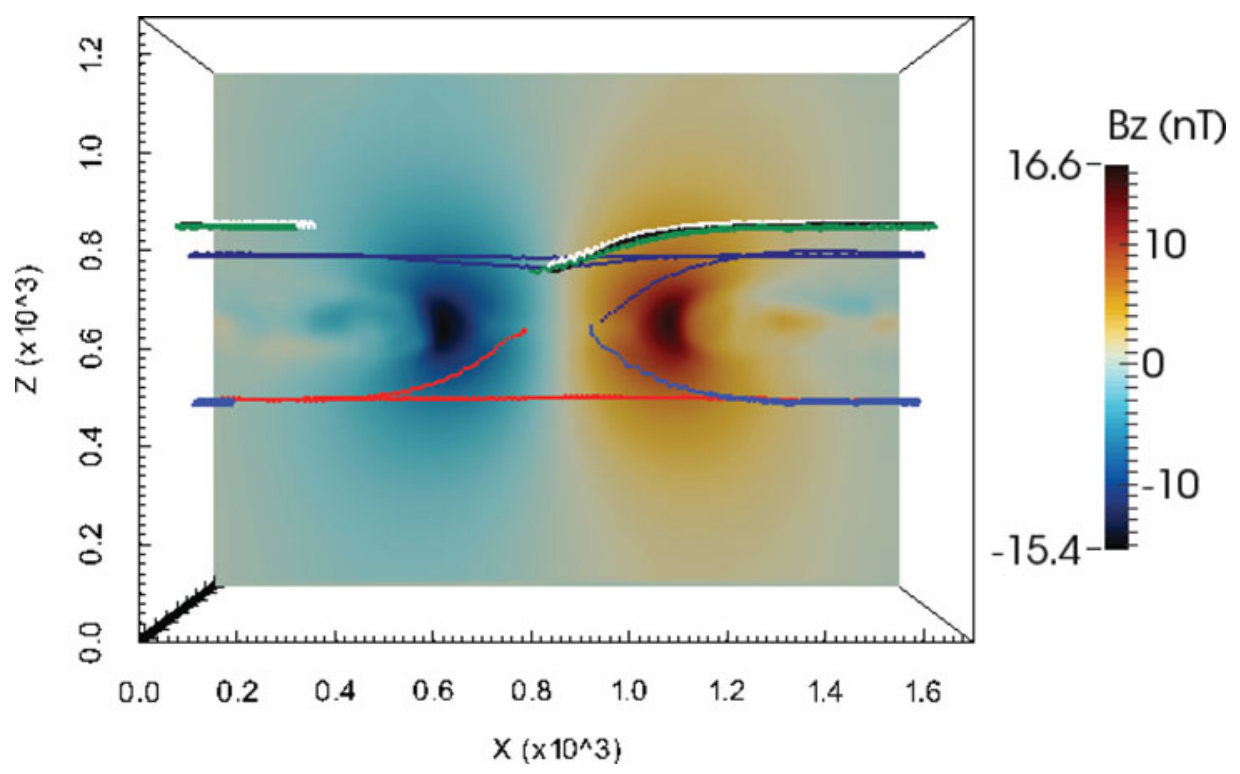

FIGURE 6. Trajectories of passing electrons in the reconnection region superimposed to the contourplot $B_{z}$ magnetic field component at time $0.2 \mathrm{~s}\left(2353 \omega_{p i}^{-1}\right)$. These electrons pass the reconnection region without being trapped.

of the reconnection fronts but not in the reconnection region, which is different from the electrons. The maximum ion velocity is approximately five times the Alfvén velocity. Two ion trajectory categories have been identified. Some of these ions cross the current sheet as the effect of $\nabla \mathbf{B}$ drift, while some others do not cross the current sheet but keep rotating along the lobe magnetic field (Speiser 1965). 

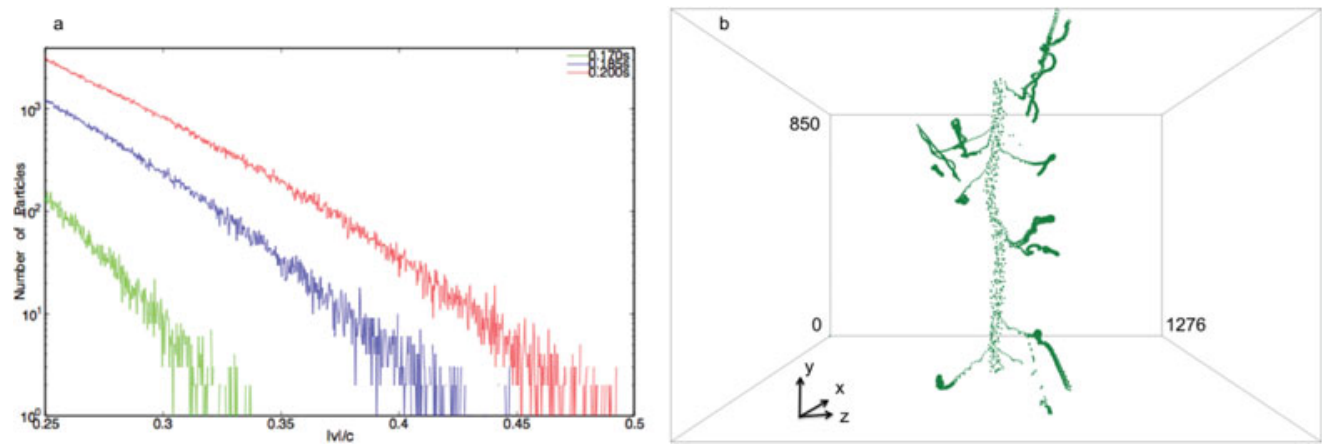

FiguRE 7. In (a) electron velocity distribution function for $|v|>54000 \mathrm{~km} \mathrm{~s}^{-1}$ at times $t=0.17,0.185,0.2 \mathrm{~s}$ are in blue, green and red respectively. The number of relativistic electrons increases during magnetic reconnection. In (b) trajectories of electrons with $|v|>1 / 2 c$ show that the highest velocity electrons are accelerated by the reconnection electric field along the $x$-line (the $y$ direction at the center of the simulation box).

\section{Discussion and conclusions}

A 3D Particle-in-Cell simulation of magnetic reconnection in the Earth's magnetotail has been carried out. The paper focuses on the study of the dynamics of energetic particles. We implemented a relativistic particle mover in the iPIC3D Particle-in-Cell code to correctly model the trajectories of high energetic particles. The relativistic mover is based on the numerical solution of the relativistic equation of motion and the predictor-corrector technique. The positions and velocities of all simulation particles have been saved at high frequency (every four computational cycles) so that particle trajectories and velocities can be reconstructed with high accuracy.

The presence of quadrupolar structure of Hall magnetic field, reconnection electric field and recognition jets is the signature of magnetic reconnection. Previous studies, presenting the results of magnetic reconnection with the presence of a guide field, are in agreement with the results obtained in our simulations without a guide field. Two-dimensional cold-fluid simulations in a configuration with strong magnetic guide field showed that electron currents locate along $x$-line and along separatrices (Ottaviani and Porcelli 1993). Previous works also show the impact from the magnitude of guide field to the localization of the reconnection jets (Fu et al. 2006) and to the peak electron peak velocity (Ricci et al. 2003).

We noticed that value of the reconnection electric field is approximately 10 times the value typically observed in magnetotail data from spacecrafts. This discrepancy is due to the use of reduced ion to electron mass ratio and consequently the $c / V_{A}$ ratio is reduced in the simulation. The reconnection fronts are affected by an instability. The presence of lower hybrid drift instability has been detected along the current sheet before the onset of magnetic reconnection. It has been shown that the lower hybrid drift instability accelerates electrons. In addition, we found that energetic electrons are localized in the reconnection region along the $x$-line and in the separatrices during magnetic reconnection. Energetic particles along separatrices surfaces first fill a smaller region and then extend to all the surfaces. Along the separatrices, regions with strong deceleration are found. In the $x$-line region, we found two categories of electron trajectories. First, part of the electrons are trapped in the reconnection region, bouncing a few times. This trapping has been discussed in Egedal et al. (2005, 2008). Second, part of the electrons pass the reconnection without being trapped. Differently 
from electrons, ions are not accelerated to high velocities. The most energetic ions are localized on the reconnection fronts.

In summary, we implemented a relativistic particle mover in the iPIC3D code and used it to model the dynamics of high energetic particles. To answer the scientific question about the dynamics and acceleration mechanism of high energetic particles in magnetic reconnection, we carried out a 3D fully kinetic simulation of magnetic reconnection in a configuration that mimics the Earth's magnetotail configuration. The main results of this work are:

(1) Energetic electrons are localized along the $x$-line and along the separatrices regions. The energetic electrons are first present in localized stripe of the separatrices and finally cover all the separatrix surfaces.

(2) Along the separatrices, electrons are decelerated to almost vanishing velocity. This is probably the result of a kinetic instability such as Buneman or two stream instability as reported in previous works (Lapenta et al. 2011; Divin et al. 2012; Markidis et al. 2012).

(3) Electrons close to the current sheet are accelerated by the lower-hybrid waves generated by lower-hybrid drift instability at the early stage of the simulation. At the late stage of the simulation, electrons are accelerated by an instability of the reconnection fronts (Vapirev et al. 2013; Lapenta et al. 2014).

(4) Few electrons $\left(<10^{-6 \%}\right)$ are accelerated to high relativistic energy by the reconnection electric field. These electrons are localized along the $x$-line and move in the $y$ direction.

(5) Two kinds of trajectories of high energetic electrons are found: trapped and passing trajectories. Some electrons are trapped in the reconnection region bouncing back from separatrices regions. These kind of trajectories were found in previous studies (Egedal et al. 2005).

(6) Ions are not accelerated to relativistic velocities. Ions with highest energy are located in the fronts of the reconnection jets.

One limitation of the proposed simulation is the use of reduced ion to electron mass ratio. Future works will focus on simulations with higher ion to electron mass ratios to assess the importance of using realistic values in 3D Particle-in-Cell simulations.

\section{Acknowledgments}

This work was funded by the Swedish VR grant D621-2013-4309 and by the European Commission through the EPiGRAM project (grant agreement no. 610598. epigram-project.eu).

\section{REFERENCES}

Ashour-Abdalla, M., El-Alaoui, M., Goldstein, M. L., Zhou, M., Schriver, D., Richard, R., Walker, R., Kivelson, M. G. and Hwang, K.-J. 2011 Observations and simulations of non-local acceleration of electrons in magnetotail magnetic reconnection events. Nature Phys. 7(4), 360-365.

Birdsall, C. K. and Langdon, A. B. 2004 Plasma Physics Via Computer Simulation. New York: CRC Press.

Birn, J., Artemyev, A. V., Baker, D. N., Echim, M., Hoshino, M. and Zelenyi, L. M. 2012 Particle acceleration in the magnetotail and aurora. Space Sci. Rev. 173(1-4), 49-102.

Birn, J. et al. 2001 Geospace environmental modeling (GEM) magnetic reconnection challenge. $J$. Geophys. Res. (Space Phys.) 106, 3715-3720.

Birn, J. and Priest, E. R. 2007 Reconnection of Magnetic Fields. Cambridge: Cambridge University Press. 
Daughton, W. 2003 Electromagnetic properties of the lower-hybrid drift instability in a thin current sheet. Phys. Plasmas 10, 3103.

Daughton, W., Roytershteyn, V., Karimabadi, H., Yin, L., Albright, B. J., Bergen, B. and Bowers, K. J. 2011 Role of electron physics in the development of turbulent magnetic reconnection in collisionless plasmas. Nature Phys. 7, 539-542.

Divin, A., Lapenta, G., Markidis, S., Newman, D. L. and Goldman, M. V. 2012 Numerical simulations of separatrix instabilities in collisionless magnetic reconnection. Phys. Plasmas (1994-present) 19(4), 042110.

Divin, A., Markidis, S., Lapenta, G., Semenov, V. S., Erkaev, N. V. and Biernat, H. K. 2010 Model of electron pressure anisotropy in the electron diffusion region of collisionless magnetic reconnection. Phys. Plasmas (1994-present) 17(12), 122102.

Egedal, J., Fox, W., Katz, N., Porkolab, M., Øieroset, M., Lin, R. P., Daughton, W. and Drake, J. F. 2008 Evidence and theory for trapped electrons in guide field magnetotail reconnection. $J$. Geophys. Res.: Space Phys. (1978-2012) DOI: 10.1029/2008JA013520.

Egedal, J., Øieroset, M., Fox, W. and Lin, R. P. 2005 In situ discovery of an electrostatic potential, trapping electrons and mediating fast reconnection in the earth's magnetotail. Phys. Rev. Lett. 94(2), 025006.

Finn, J. M. 2006 Magnetic reconnection: null point. Nature Phys. 2(7), 445-446.

$\mathrm{Fu}, \mathrm{X}$. R., Lu, Q. M. and Wang, S. 2006 The process of electron acceleration during collisionless magnetic reconnection. Phys. Plasmas (1994-present) 13(1), 012309.

Hockney, R. W. and Eastwood, J. W. 1988 Computer Simulation using Particles. New York: CRC Press.

Hoshino, M. 2005 Electron surfing acceleration in magnetic reconnection. J. Geophys. Res.: Space Phys. (1978-2012) DOI: 10.1029/2005JA011229.

Hoshino, M., Mukai, T., Terasawa, T. and Shinohara, I. 2001 Suprathermal electron acceleration in magnetic reconnection. J. Geophys. Res.: Space Phys. (1978-2012) 106(A11), 25 979-25997.

Kivelson, M. G. and Russell, C. T. 1995 Introduction to Space Physics. Cambridge: Cambridge University Press.

Lapenta, G., Brackbill, J. U. and Daughton, W. S. 2003 The unexpected role of the lower hybrid drift instability in magnetic reconnection in three dimensions. Phys. Plasmas 10(5), $1577-$ 1587.

Lapenta, G., Brackbill, J. U. and Ricci, P. 2006 Kinetic approach to microscopic macroscopic coupling in space and laboratory plasmas. Phys. Plasmas 13(5), 055904.

Lapenta, G., Goldman, M., Newman, D., Markidis, S. and Divin, A. 2014 Electromagnetic energy conversion in downstream fronts from three dimensional kinetic reconnection. Phys. Plasmas (1994-present) 21(5), 055702.

Lapenta, G., Markidis, S., Divin, A., Goldman, M. and Newman, D. 2010 Scales of guide field reconnection at the hydrogen mass ratio. Phys. Plasmas (1994-present) 17(8), 082106.

Lapenta, G., Markidis, S., Divin, A., Goldman, M. V. and Newman, D. L. 2011 Bipolar electric field signatures of reconnection separatrices for a hydrogen plasma at realistic guide fields. Geophys. Res. Lett. 38 L17 104.

Loureiro, N. F., Schekochihin, A. A. and Zocco, A. 2013 Fast collisionless reconnection and electron heating in strongly magnetized plasmas. Phys. Rev. Lett. 111(2), 025002.

Markidis, S., Henri, P., Lapenta, G., Divin, A., Goldman, M. V., Newman, D. and Eriksson, S. 2012 Collisionless magnetic reconnection in a plasmoid chain. Nonlinear Process. Geophys. 19(1), $145-153$.

Markidis, S., Lapenta, G. and Rizwan-uddin 2010 Multi-scale simulations of plasma with iPIC3D. Math. Comput. Simul. 80(7), 1509-1519.

Nagai, T., Shinohara, I., Fujimoto, M., Hoshino, M., Saito, Y., Machida, S. and Mukai, T. 2001 Geotail observations of the hall current system: evidence of magnetic reconnection in the magnetotail. J. Geophys. Res.: Space Phys. 106 (A11), 25 929-25949.

Nagai, T., I. Shinohara, S. Zenitani, R. Nakamura, T. K. M. Nakamura, M. Fujimoto, Y. Saito, and T. Mukai. "Three-dimensional structure of magnetic reconnection in the magnetotail from Geotail observations." Journal of Geophysical Research: Space Physics 118, no. 4 (2013): $1667-1678$.

Noguchi, K., Tronci, C., Zuccaro, G. and Lapenta, G. 2007 Formulation of the relativistic moment implicit particle-in-cell method. Phys. Plasmas (1994-present) 14(4), 042308. 
Øieroset, M., Lin, R. P., Phan, T. D., Larson, D. E. and Bale, S. D. 2002a Evidence for electron acceleration up to $300 \mathrm{keV}$ in the magnetic reconnection diffusion region of earth's magnetotail. Phys. Rev. Lett. 89, 195001.

Øieroset, M., Lin, R. P., Phan, T. D., Larson, D. E. and Bale, S. D. 2002b Evidence for electron acceleration up to $300 \mathrm{kev}$ in the magnetic reconnection diffusion region of Earth's magnetotail. Phys. Rev. Lett. 89(19), 195001.

Ottaviani, M. and Porcelli, F. 1993 Nonlinear collisionless magnetic reconnection. Phys. Rev. Lett. 71(23), 3802.

Pegoraro, F., Borgogno, D., Califano, F., Sarto, D. D., Echkina, E., Grasso, D., Liseikina, T. and Porcelli, F. 2004 Developments in the theory of collisionless reconnection in magnetic configurations with a strong guide field. Nonlinear Process. Geophys. 11(5/6), 567-577.

Press, W. H. 2007 Numerical Recipes: The Art of Scientific Computing, 3rd edn. Cambridge: Cambridge University Press.

Priest, E. and Forbes, T. 2007 Magnetic reconnection: MHD theory and applications. Cambridge: Cambridge University Press.

Retinò, A. et al. 2008 Cluster observations of energetic electrons and electromagnetic fields within a reconnecting thin current sheet in the earth's magnetotail. J. Geophys. Res.: Space Phys. (1978-2012) DOI: 10.1029/2008JA013511.

Ricci, P., Brackbill, J. U., Daughton, W. and Lapenta, G. 2005 New role of the lower-hybrid drift instability in the magnetic reconnection. Phys. Plasmas 12(5), 055901.

Ricci, P., Lapenta, G. and Brackbill, J. U. 2003 Electron acceleration and heating in collisionless magnetic reconnection. Phys. Plasmas (1994-present) 10(9), 3554-3560.

Russell, C. T. 1971 Geophysical coordinate transformations. Cosm. Electrodyn. 2(2), 184-196.

Sharma, A. S. et al. 2008 Transient and localized processes in the magnetotail: a review. In Ann. Geophys. 26, 955-1006. Göttingen.

Sonnerup, B. U. 1974 Magnetopause reconnection rate. J. Geophys. Res. 79(10), 1546-1549.

Speiser, T. W. 1965 Particle trajectories in model current sheets: 1. Analytical solutions. J. Geophys. Res. 70(17), 4219-4226.

van der Plas, E. V. and de Blank, H. J. 2007 Temperature gradients in fast collisionless magnetic reconnection. Phys. Rev. Lett. 98(26), 265002.

Vapirev, A. E., Lapenta, G., Divin, A., Markidis, S., Henri, P., Goldman, M. and Newman, D. 2013 Formation of a transient front structure near reconnection point in 3-D pic simulations. $J$. Geophys. Res.: Space Phy. 118 (4), 1435-1449.

Vay, J.-L. 2008 Simulation of beams or plasmas crossing at relativistic velocity. Phys. Plasmas (1994-present) 15(5), 056701. 